\section{Lack of benefit of early awareness to age-related macular degeneration}

RA Cervantes-Castañeda, E Banin, I Hemo, M Shpigel, E Averbukh and I Chowers

\begin{abstract}
Aims To assess the rate of early awareness to the presence of age-related macular degeneration (AMD) and whether it enables early detection of transition to neovascular AMD (NVAMD) as compared with patients whose first presentation to an ophthalmologist is already at the neovascular stage of disease. Methods A retrospective analysis of 268 eyes of 268 consecutive patients with newly diagnosed NVAMD that were treated in a tertiary referral centre was performed. Patients were classified into those who were unaware (Group 1), or aware (Group 2), of the fact that they had AMD before diagnosis of NVAMD.

Visual acuity, lesion size and composition, and demographics were compared between both groups.

Results In all, 185 patients (69\%) and 83 patients (31\%) were classified to Groups 1 and 2 , respectively. Patients in Groups 1 and 2 had similar demographic characteristics, presenting visual acuity and lesion size, and lesion compositions. Group 1 patients were more likely to have a positive history for smoking (41 vs $26 \%$ in Group 2, $P=0.03$ ), whereas Group 2 patients were more likely to have positive family history for AMD (20 vs 10\%, $P=0.02$ ).

Conclusions These data suggest that current screening methods fail to identify the majority of patients with AMD before the development of NVAMD. The findings also demonstrate that in the routine clinical setting, prior awareness of AMD may not facilitate early detection of treatable choroidal neovascularization lesions.
\end{abstract}

Eye (2008) 22, 777-781; doi:10.1038/sj.eye.6702691; published online 12 January 2007

Keywords: age-related macular degeneration; early awareness; visual acuity; lesion size
Introduction

Patients who develop choroidal neovascularization ( $\mathrm{CNV}$ ) secondary to age-related macular degeneration (AMD) usually present with significant visual loss. For example, Olsen et al. ${ }^{1}$ found that $78.5 \%$ of their patients presenting with neovascular AMD (NVAMD) had visual acuity of $20 / 50$ or worse. Majority of such patients fail to achieve substantial visual recovery despite the application of current therapies. ${ }^{2-5}$

A number of lines of evidence suggest that earlier detection of CNV may confer a better prognosis. As such, smaller initial lesion size is associated with better visual outcome following photodynamic therapy (PDT) for NVAMD. ${ }^{6}$ Furthermore, as reduction of the risk for three-line loss following PDT is not significantly dependent on the presenting visual acuity, better initial visual acuity before PDT may confer better final acuity following the therapy. Although such data is not yet available for novel antivascular endothelial growth factor (VEGF)-based therapies such as intravitreal injections of Bavacizumab or Ranibizumab, these facts underscore the importance of early detection of CNV. Such early diagnosis may enable detection of patients with relatively small lesions, preserved visual acuity, and juxtafoveal and extrafoveal lesions. ${ }^{7,8}$

To facilitate early detection of $\mathrm{CNV}$, current practice guidelines for patients with intermediate and monocular advanced AMD mandate periodic follow-up, self-test with an Amsler grid, and prompt examination by an ophthalmologist once new symptoms such as metamorphopsia, visual loss, or scotomas occur. ${ }^{9}$ Prior diagnosis of non-NVAMD is obviously a prerequisite to allow initiation of such a follow-up regimen and it also allows for commencement of oral supplement therapy according to the AREDS study recommendations. ${ }^{10}$
Department of

Ophthalmology, Hadassah Hebrew University Medical Center, Jerusalem, Israel

Correspondence: I Chowers, Department of

Ophthalmology,

Hadassah - Hebrew

University Medical Center, PO Box 12000,

Ein Kerem,

Jerusalem 91120, Israel

Tel: + 9722 6777882;

Fax: +972 26428896.

E-mail: chowers@

md.huji.ac.il

Received: 15 June 2006 Accepted in revised form: 17 November 2006 Published online: 12 January 2007 
The current practice guidelines for patients with intermediate AMD or monocular advanced AMD are based on extrapolations from studies suggesting benefit for early detection of $\mathrm{CNV}$, and on the potential value of the Amsler grid in facilitating such early diagnosis. ${ }^{9}$ Interestingly, the value and efficacy of this follow-up regimen for enabling early detection and improving visual outcome has not been well documented thus far. Furthermore, the percentage of AMD patients who are diagnosed before the development of $\mathrm{CNV}$ is unknown. Knowing the efficiency of screening practice for AMD is important to improve treatment algorithm of AMD.

To begin and address these issues, we measured the percentage of patients presenting with NVAMD to a tertiary referral centre who were aware of the fact that they had AMD before the development of CNV. We then assessed whether such prior awareness enabled early detection of CNV.

\section{Methods}

In total, 268 consecutive patients (268 study eyes), who were diagnosed with NVAMD and treated with PDT at the Department of Ophthalmology of the Hadassah Hebrew University Medical Center in Jerusalem, Israel, from November 2004 through June 2006, were included in the study. During the study period, this was the only centre that provided PDT for residents of Jerusalem. Institutional Ethics Committee approval was obtained for the study. Retrospective data were collected on patients who also had PDT sessions before the study starting date. AMD was diagnosed and graded as described in the AREDS trial. ${ }^{10}$ Each patient was interviewed, patients' charts were reviewed, and a detailed ophthalmic exam was performed which also included best-corrected visual acuity assessment using an ETDRS chart. Fluorescein angiograms were reviewed by a retina specialist (IC, EB, $\mathrm{IH}$, and EA). The standard PDT protocol for NVAMD was applied using intravenous infusion of verteporfin (Visudyne; Novartis Ophthalmics, Hettlingen,
Switzerland) followed by diode laser application (Visulas s 690, Zeiss, Switzerland) using the PDT laser lens (Volk, Mentor, OH, USA). Patients were then followed at 3month intervals. ${ }^{5}$ Repeated PDT sessions were performed according to accepted guidelines. ${ }^{11}$

Patients were classified into those who were not aware of being previously diagnosed with AMD and had presented to an ophthalmologist only after they have developed CNV in the study eye (Group $1=$ unaware patients), and patients who were aware that they had AMD before the development of CNV in the study eye (Group 2 =aware patients). Patients were classified as aware if: (1) they recalled that they were previously diagnosed with a degeneration of the retina or macula and/or (2) patient recalled that oral supplementation according to the AREDS trial recommendations was prescribed, and/or (3) patient recalled that the use of an Amsler grid was suggested by an ophthalmologist. As one of the major aims of the study was to assess if prior diagnosis of AMD confers overall better outcome in the normal clinical setting (rather than a study environment), patients were not required to be familiar with the term AMD in order to be classified as aware (given that at least one of the three conditions listed above applied for the patient). Statistical analysis was performed using $t$-test, Mann-Whitney test, and the Fisher's exact test.

\section{Results}

Of the 268 patients included in the study, 185 (69\%) were classified as belonging to Group 1 (previously unaware of having AMD) whereas 83 patients (31\%) were classified to Group 2 (aware). Patients in the two groups had similar demographic characteristics except for history of smoking and family history of AMD (Table 1). Although $41 \%$ of the patients in Group 1 had a positive smoking history, only $26 \%$ of the patients in Group 2 were smokers or past smokers $(P=0.03$, Fisher's Exact test). A higher percentage of patients in Group $2(20 \%)$ compared with Group $1(10 \%)$ had additional family

Table 1 Patients demographics

\begin{tabular}{lccc}
\hline & Group 1 (unaware) & Group 2 (aware) & P-value* \\
\hline Number of patients & 185 & 83 & NA \\
Male/female & $104 / 81$ & $44 / 39$ & 0.6 \\
Age (median/range, in years) & $79 / 57-92$ & $79 / 64-92$ & 0.2 \\
Smoking (\%) & $76(41 \%)$ & $22(26 \%)$ & 0.03 \\
Family history of AMD (\%) & $18(10 \%)$ & $17(20 \%)$ & 0.02 \\
Systemic hypertension (\%) & $110(61 \%)$ & $45(54 \%)$ & 0.4 \\
Follow-up period (median, range; in months) & $18,1-137$ & $14,1-216$ & 0.7 \\
Study eye (right/left) & $91 / 94$ & $32 / 51$ & 0.1 \\
\hline
\end{tabular}

NA, not applicable.

${ }^{*} P$-value calculated using Fisher's exact test (awareness, gender, smoking, family history, hypertension, eye laterality), or $t$-test (age, follow-up period). 
members who were affected by $\operatorname{AMD}(P=0.02$, Fisher's exact test).

Frequency of different angiographic lesion compositions at diagnosis (occult, minimally classic, or predominantly classic) was similar between both groups (Table 2). Mean initial and final lesion size, initial and final visual acuity, follow-up time, and number of PDT sessions were also similar between the two groups (Tables 1-3).

There was a nonsignificant trend towards better initial visual acuity in the fellow eyes of patients from Group 1 (mean LogMAR visual acuity of 0.52 or approximately 0.32 ETDRS acuity) compared with patients from Group 2 (mean Log MAR visual acuity of 0.76 or approximately 0.16-0.2 ETDRS acuity) (Table 3).

Intuitively, one may assume that patients with significantly decreased vision in one eye would be more apt to detect early reduction of visual acuity in the contra-lateral better eye once it develops CNV. To assess this possibility, we tested the correlation between fellow eye and study eye initial visual acuities. We found that in either group, fellow eye visual acuity did not directly or inversely correlate with visual acuity of the study eye (Group 1: $r^{2}=0.001, P=0.67$; Group 2: $r^{2}=0.01, P=0.47$, linear regression test). Thus, in contrast to what might be intuitively presumed, low visual acuity in the fellow eye did not confer an increased probability for early detection of decreased vision in the study eye.

\section{Discussion}

In our cohort from a tertiary referral centre, the majority of patients who presented with NVAMD and who were treated with PDT were unaware that they had AMD before the diagnosis of CNV. Among those who were aware, early awareness of the disease did not confer earlier detection of the transition to NVAMD in the study eye nor better outcome in terms of visual acuity, lesion size, or lesion composition in patients treated with PDT. These findings point towards two major limitations of the current clinical management practice of AMD patients.
First, many patients with early and intermediate AMD escape diagnosis until they develop CNV and visual loss. Some patients with monocular advanced AMD remain undiagnosed until they develop CNV in the fellow eye. Such undiagnosed AMD patients do not enjoy the benefit of oral vitamin and mineral supplementation according to the AREDS recommendations. These patients also miss the potential for early detection of $\mathrm{CNV}$ that may perhaps be obtained by regular follow-up and self-examination regimens.

This apparent failure to diagnose AMD before the development of $\mathrm{CNV}$ can be attributed to low rates of routine screening exams by ophthalmologists, and potentially the failure to identify AMD during such exams. Inadequate transfer of information and education of patients may also be the underlying cause in at least some cases (in other words, the ophthalmologist has perhaps identified signs of AMD but failed to transfer this knowledge effectively to the patient). A recent survey commissioned by the AMD Alliance International in Western European countries, Australia, Israel, and the United States reported that between 58 and $77 \%$ of participants had an eye exam during the preceding 2 years (http:/ /www.amdalliance.org/, and a country report by AMD Alliance in Israel, 2004). Although many such eye exams might not include a dilated fundus exam, these data suggest that a significant percentage of undiagnosed AMD patients may be owing to missed diagnosis by ophthalmologists, or a failure in informing

Table 3 Visual acuity parameters

\begin{tabular}{lccc}
\hline & $\begin{array}{c}\text { Group 1 } \\
\text { (unaware) }\end{array}$ & $\begin{array}{c}\text { Group 2 } \\
\text { (aware) }\end{array}$ & P-value* \\
\hline Initial study eye VA & $1.03 \pm 0.76$ & $1.1 \pm 0.83$ & 0.4 \\
Final study eye VA & $1.28 \pm 0.8$ & $1.22 \pm 0.6$ & 0.6 \\
Initial fellow eye VA & $0.52 \pm 0.73$ & $0.76 \pm 0.85$ & 0.08 \\
Final fellow eye VA & $1 \pm 1$ & $1 \pm 0.92$ & 0.5 \\
\hline
\end{tabular}

VA, visual acuity.

Best-corrected visual acuity measured with an ETDRS chart. All results presented as mean $\log \mathrm{MAR} \pm \mathrm{SD}$; *analysis was performed using the Mann-Whitney nonparametric test.

Table 2 Lesion and treatment characteristics

\begin{tabular}{lccc}
\hline & Group 1 (unaware) & Group 2 (aware) & P-value* $^{*}$ \\
\hline Occult $(\%)$ & 35 & 45 & 0.12 \\
Predominantly classic (\%) & 61 & 51 & 0.44 \\
Minimally classic (\%) & 4 & 4 & 0.9 \\
Initial lesion GLD (mean \pm SD, in $\mu \mathrm{m}$ ) & $3700 \pm 1275$ & $3650 \pm 1150$ & 0.8 \\
Final lesion GLD (mean \pm SD, in $\mu \mathrm{m}$ ) & $4025 \pm 1265$ & $4070 \pm 1165$ & 0.86 \\
Number of PDT sessions (mean \pm SD) & $2.8 \pm 1.7$ & $2.8 \pm 1.7$ & 0.72 \\
\hline
\end{tabular}

GLD, greater linear diameter; PDT, photodynamic therapy.

*Statistical analysis of lesions type was performed using Fisher's exact test; lesion size and PDT treatment session number were compared using $t$-test. 
and educating patients about their disease. Considering the wide availability of ophthalmologists in Israel, the national health care system which essentially covers the cost of eye exams, and the small size of the country, it is unlikely that difficulties in gaining access to an ophthalmologist play a major role in these high rates of under-diagnosed AMD.

We found that 'aware' patients were less likely to be smokers but had higher probability of a positive family history of AMD. These factors may reflect overall higher personal awareness to the importance of preventative medicine (avoidance of smoking). It may also demonstrate that familiarity with the disorder (having seen its impact on a family member) perhaps increases the likelihood for seeking it out and being aware of the disorder in oneself.

A second limitation of current management of intermediate and monocular advanced AMD, which is highlighted by our research, is the failure of prior awareness to the disease to facilitate early detection of $\mathrm{CNV}$. Missing scheduled follow-up exams, inappropriate use of the Amsler grid (or perhaps low efficacy of this screening test), delay in approaching an ophthalmologist despite the appearance of new symptoms, and delay in administration of appropriate treatment after initial diagnosis of new $\mathrm{CNV}$ may all contribute to this failure.

It is important to note that as the majority of patients in our cohort were referred by primary ophthalmologists in the community, it was not possible to reliably assess adherence to recommended practice guidelines. It is possible that strict adherence to such guidelines may confer better outcome than that experienced by the patients in this cohort. Few studies have assessed adherence of AMD patients to the recommended follow-up guidelines. Lee $e t$ al. ${ }^{12}$ reported that among patients with AMD, 65-80\% had annual eye examinations, and the same group also showed that several factors, among them presence of NVAMD, income, education, race, and availability of eye care, are associated with the likelihood of compliance with practice guidelines. ${ }^{13}$

Although poor compliance with practice guidelines may have significantly contributed to the low rate of early detection of $\mathrm{CNV}$, several lines of evidence suggest that current guidelines might be suboptimal for obtaining such early detection. Importantly, the optimal interval between follow-up visits for patients with intermediate AMD and monocular advanced AMD is unknown. An interval of 6-12 months was suggested in the preferred practice guidelines issued by the American Academy of Ophthalmology in 2003. ${ }^{9}$ However, CNV growth rate may reach $74 \mu \mathrm{m}$ per day with an average growth rate of between 10 and $18 \mu \mathrm{m}$ per day. ${ }^{14,15}$ Therefore, periodic follow-up every 6 months or more might allow CNV to reach considerable size before diagnosis. Accordingly, some ophthalmologists prefer to evaluate such patients more frequently.

Self test with an Amsler grid might also be insufficient to facilitate early detection of CNV. Although the use of Amsler grids for monitoring the central visual field for the appearance of new scotomas and metamorphopsia has been recommended for AMD patients ${ }^{9,16}$ studies have demonstrated that the sensitivity for detecting AMD lesions may be low. ${ }^{17-20}$ Hopefully, novel diagnostic modalities such as the preferential hyperacuity perimeter or others will have higher sensitivity and specificity for this purpose. ${ }^{21-23}$

The most significant caveat of our study is its single tertiary centre design. Although such design may potentially introduce bias, such bias is probably minimized by the referral pattern in our region. Our centre was the only PDT facility for Jerusalem residence during the study period. As PDT was the only available treatment for NVAMD through most of the study period, patients who develop NVAMD but who were not referred to receive PDT in our centre experienced the natural course of the disease. Awareness to the fact that they had AMD before developing CNV could not affect the outcome of such patients who were not included in our study.

In light of the magnitude of the challenge posed by AMD and the apparent potential limitations of current screening and follow-up practices, it is imperative to evaluate in a prospective manner and to modify accordingly these guidelines to allow for higher rates of early detection of AMD and CNV. Better education of both patients and physicians, alterations in follow-up intervals, and introduction of novel screening modalities, may all be required to achieve this goal.

\section{Acknowledgements}

This work was presented in part in the ARVO annual meeting, Fort Lauderdale, FL, USA, 30 April-4 May 2006.

\section{References}

1 Olsen TW, Feng X, Kasper TJ, Rath PP, Steuer ER. Fluorescein angiographic lesion type frequency in neovascular age-related macular degeneration. Ophthalmology 2004; 111: 250-255.

2 Gragoudas ES, Adamis AP, Cunningham Jr ET, Feinsod M, Guyer DR. Pegaptanib for neovascular age-related macular degeneration. N Engl J Med 2004; 351: 2805-2816.

3 Bressler NM. Photodynamic therapy of subfoveal choroidal neovascularization in age-related macular degeneration with verteporfin: two-year results of 2 randomized clinical trials-tap report 2. Arch Ophthalmol 2001; 119: 198-207. 
4 Rosenfeld PJ, Moshfeghi AA, Puliafito CA. Optical coherence tomography findings after an intravitreal injection of bevacizumab (avastin) for neovascular agerelated macular degeneration. Ophthalmic Surg Lasers Imaging 2005; 36: 331-335.

5 Avery RL, Pieramici DJ, Rabena MD, Castellarin AA, Nasir MA, Giust MJ. Intravitreal bevacizumab (Avastin) for neovascular age-related macular degeneration. Ophthalmology 2006; 113: 363-372.

6 Blinder KJ, Bradley S, Bressler NM, Bressler SB, Donati G, Hao Y et al. Effect of lesion size, visual acuity, and lesion composition on visual acuity change with and without verteporfin therapy for choroidal neovascularization secondary to age-related macular degeneration: TAP and VIP report no. 1. Am J Ophthalmol 2003; 136: 407-418.

7 Grey RH, Bird AC, Chisholm IH. Senile disciform macular degeneration: features indicating suitability for photocoagulation. Br J Ophthalmol 1979; 63: 85-89.

8 Bressler NM. Early detection and treatment of neovascular age-related macular degeneration. J Am Board Fam Pract 2002; 15: 142-152

9 Preferred practice pattern: age related macular degeneration. American Academy of Ophthalmology: San Francisco, USA, 2003.

10 A randomized placebo-controlled clinical trial of high-dose supplementation with vitamins $\mathrm{C}$ and $\mathrm{E}$ beta carotenezinc for age-related macular degeneration vision loss: AREDS report no. 8. Arch Ophthalmol 2001; 119: 1417-1436.

11 Guidelines for using verteporfin (Visudyne) in photodynamic therapy for choroidal neovascularization due to age-related macular degeneration other causes: update. Retina 2005; 25: 119-134.

12 Lee PP, Feldman ZW, Ostermann J, Brown DS, Sloan FA. Longitudinal rates of annual eye examinations of persons with diabetes and chronic eye diseases. Ophthalmology 2003; 110: 1952-1959.

13 Sloan FA, Brown DS, Carlisle ES, Picone GA, Lee PP. Monitoring visual status: why patients do or do not comply with practice guidelines. Health Serv Res 2004; 39: 1429-1448.

14 Klein ML, Jorizzo PA, Watzke RC. Growth features of choroidal neovascular membranes in age-related macular degeneration. Ophthalmology 1989; 96: 1416-1419.

15 Vander JF, Morgan CM, Schatz H. Growth rate of subretinal neovascularization in age-related macular degeneration. Ophthalmology 1989; 96: 1422-1426; discussion 6-9.

16 Fine SL. Early detection of extrafoveal neovascular membranes by daily central field evaluation. Ophthalmology 1985; 92: 603-609.

17 Loewenstein A, Malach R, Goldstein M, Leibovitch I, Barak A, Baruch E et al. Replacing the Amsler grid: a new method for monitoring patients with age-related macular degeneration. Ophthalmology 2003; 110: 966-970.

18 Roy MS. Vision loss without Amsler grid abnormalities in macular subretinal neovascularization. Ophthalmologica 1985; 191: 215-217.

19 Achard OA, Safran AB, Duret FC, Ragama E. Role of the completion phenomenon in the evaluation of Amsler grid results. Am J Ophthalmol 1995; 120: 322-329.

20 Zaidi FH, Cheong-Leen R, Gair EJ, Weir R, Sharkawi E, Lee $\mathrm{N}$ et al. The Amsler chart is of doubtful value in retinal screening for early laser therapy of subretinal membranes. The West London Survey. Eye 2004; 18: 503-508.

21 Arden GB, Wolf JE. Colour vision testing as an aid to diagnosis and management of age related maculopathy. $\mathrm{Br} \mathrm{J}$ Ophthalmol 2004; 88: 1180-1185.

22 Fink W, Sadun AA. Three-dimensional computerautomated threshold Amsler grid test. J Biomed Opt 2004; 9: 149-153.

23 Goldstein M, Loewenstein A, Barak A, Pollack A, Bukelman A, Katz $\mathrm{H}$ et al. Results of a multicenter clinical trial to evaluate the preferential hyperacuity perimeter for detection of age-related macular degeneration. Retina 2005; 25: 296-303. 